\title{
INFLUENCIA DO USO DE LÍQUIDOS IÔNICOS NO PROCESSO DE IMOBILIZAÇÃO DE LIPASE BACILLUS SP. EM BIOPOLÍMERO
}

\author{
E. B. M. FILHO ${ }^{1}$, A. T. FRICKS ${ }^{1,2}$, E. FRANCESCHI ${ }^{1,2}$, A. S. LIMA $^{1,2}$, D. P. SILVA ${ }^{3}$, C. \\ M. F. SOARES ${ }^{1,2}$, R. Y. CABRERA-PADILLA ${ }^{1,2}$, \\ ${ }^{1}$ Universidade Tiradentes, Programa de Pós-graduação de Engenharia de Processos \\ ${ }^{2}$ Instituto de Tecnologia e Pesquisa \\ ${ }^{3}$ Universidade Federal de Sergipe, Departamento de engenharia de produção
}

\begin{abstract}
RESUMO - Um dos grandes desafios no processo de imobilização é o uso de aditivos com objetivo de aumentar a atividade e estabilidade de biocatalisadores imobilizados, como por exemplo, o uso de líquidos iônicos. Deste modo o objetivo deste estudo foi avaliar a influência da familia catiônica $\left(\left[\mathrm{C}_{4} \mathrm{~min}\right] \mathrm{Cl}\right.$, $\left(\left[\mathrm{C}_{4} \mathrm{mpy}\right] \mathrm{Cl}\right)$, dos ânions $\left(\left[\mathrm{C}_{4} \mathrm{~min}\right] \mathrm{Cl},\left[\mathrm{C}_{4} \mathrm{~min}\right] \mathrm{N}(\mathrm{CN})_{2},\left[\mathrm{C}_{4} \mathrm{~min}\right] \mathrm{Tf}_{2} \mathrm{~N}\right)$, e do comprimento da cadeia do cátion $\left(\left[\mathrm{C}_{2} \mathrm{~min}\right] \mathrm{Tf}_{2} \mathrm{~N},\left[\mathrm{C}_{4} \mathrm{~min}\right] \mathrm{Tf}_{2} \mathrm{~N}\right)$ do líquido iônico (LI) no processo de imobilização por adsorção física utilizando a lipase Bacillus sp. (ITP-001) em poli(3-hidroxibutirato-co-hidroxivalerato) (PHBV). A recuperação de atividade (RA) e as propriedades bioquímicas do biocatalisador imobilizado (BI) mais eficiente foram determinadas por hidrólise. A maior RA do BI na avaliação da familia catiônica foi de $37 \%$ para o cátion $\mathrm{C}_{4}$ min. $\mathrm{Na}$ avaliação dos ânions se obteve a maior RA para o ânion mais hidrofóbico $\mathrm{Tf}_{2} \mathrm{~N}$. O aumento do comprimento da cadeia do cátion permitiu um aumento na RA de 40 para $56 \%$. A RA mais eficiente foi $56 \%$ utilizando o líquido iônico $\left[\mathrm{C}_{4} \mathrm{~min}\right] \mathrm{Tf}_{2} \mathrm{~N}$, cerca de duas vezes maior a RA do BI sem LI $(\mathrm{RA}=25 \%)$. Para o BI mais eficiente obtido neste estudo foi determinado o $\mathrm{pH}$ e temperatura ótimos $\left(4 \mathrm{e} 37^{\circ} \mathrm{C}\right.$ respectivamente). Os valores das constantes cinéticas foram $\mathrm{K}_{\mathrm{m}}=73,5 \mathrm{mM} \mathrm{e} \mathrm{V}_{\max }$ $=253,5 \mathrm{U} / \mathrm{g}$; e o tempo de meia-vida foi de $74,85 \mathrm{~h}$.
\end{abstract}

\section{INTRODUÇÃO}

As Bacillus lipases pertecem a uma ampla e diversa família de enzimas, que podem ser facilmente produzidos e tem demonstrado sua versatilidade, especificidade e estabilidade, bem como a tolerância a solventes, sais e detergentes. Portanto, Bacillus lipases pode, potencialmente, ser aplicado na indústria de alimentos, formulações de lavanderia, indústria de papel e couro, tratamento de águas residuais, e outros (Guncheva and Zhiryakova., 2011).

Lipases imobilizadas comercialmente disponíveis são, em geral, imobilizadas por adsorção física em matrizes orgânicas. Estes biocatalisadores têm custos elevados, principalmente associados ao preço do suporte (Mendes et al., 2012). Portanto, o uso de suportes mais baratos na imobilização da lipase tem sido proposto como uma alternativa aos 
suportes caros para outras aplicações em processos de grande escala. Como suporte hidrofóbico, polihidroxibutirato (PHB) foi usado para a imobilização de cinco lipases microbianas preparadas a partir de várias fontes, e usado para a hidrólise enzimática, estérificação e transesterificação (Mendes et al., 2012). Como suporte hidrofóbico promissor, o poli(hidroxibutirato-co-valerato) (PHBV) tem sido utilizado para a imobilização de lipase Cândida rugosa (Cabrera-Padilla et al., 2012) e da lipase a partir de uma nova fonte de Bacillus sp. ITP-001(Cabrera-Padilla et al., 2013), com rendimentos de imobilização de 30\% e $25 \%$, respectivamente, com razoável estabilidade térmica e operacional para ambos os biocatalizadores.

Uma forma de melhorar a atividade catalítica do biocatalisador imobilizado pode ser usando líquidos iônicos como aditivos no processo de imobilização. Estudos de vários aditivos, tais como os líquidos iônicos (LI), foram recentemente sugeridos como agentes que podem estabilizar as enzimas, protegendo a camada de hidratação ao redor da enzima e/ou causando alterações conformacionais que conduzem à ativação permanente da enzima (Hara et al., 2010). Lee et al. (2007) relataram que o processo de imobilização da lipase Cândida rugosa utilizando a técnica sol-gel mostrou uma elevada estabilidade e aumento da atividade da enzima em cerca de 10 vezes maior do que a lipase na forma livre. A influência positiva da utilização de LIs apróticos também foi relatado por Zarcula et al. (2010) utilizando [ $\left.\mathrm{C}_{8} \mathrm{mim}\right]$ $\left[\mathrm{BF}_{4}\right]$ como aditivo no processo de imobilização da lipase de Pseudomonas fluorescens em matrizes híbridas de sol-gel, os resultados obtidos com o biocatalisador imobilizado mostrou rendimento total, geralmente superior a 100 .

O objetivo deste trabalho foi imobilizar a lipase a partir de Bacillus sp. (ITP-001) por adsorção física em PHBV utilizando diferentes líquidos iônicos como aditivos do processo de imobilização, de modo a avaliar a influência da familia catiônica, dos ânions e comprimento da cadeia catiônica dos líquidos iônicos na reação de hidrólise. Foram avaliados o rendimento total da atividade recuperada e propriedades bioquímicas do biocatalisador imobilizado mais eficiente.

\section{MATERIAL E MÉTODOS}

\subsection{Obtenção da lipase purificada}

A linhagem de Bacillus sp. (ITP-001) foi isolada de um solo contaminado por petróleo da cidade de Carmópolis, localizada no estado de Sergipe, Brasil, e mantida sob refrigeração em tubos inclinados com meio YPD composto por $(\%, \mathrm{p} / \mathrm{v})$ : glicose $(2,0)$, extrato de levedura $(1,0)$, peptona $(1,0)$ e Agar $(2,5)$.

A fermentação submersa foi conduzida à $37^{\circ} \mathrm{C}$ e $170 \mathrm{rpm}$, em Erlenmeyer de $250 \mathrm{ml}$ contendo $100 \mathrm{ml}$ de meio de cultura, ajustado à $\mathrm{pH} 7 \mathrm{com} \mathrm{NaOH} 0,1 \mathrm{M}$ e $\mathrm{HCl} 0,1 \mathrm{M}$, composto por $(\%, \mathrm{~m} / \mathrm{v}): \mathrm{KH}_{2} \mathrm{PO}_{4}(0,1), \mathrm{MgSO}_{4} \cdot 7 \mathrm{H}_{2} \mathrm{O}(0,05), \mathrm{NaNO}_{3}(0,3)$, extrato de levedura $(0,6)$, peptona $(0,13)$, amido $(2,0)$, Triton X-100 $(1,0)$ e óleo de coco $(4,0)$, acrescido após 72 horas de fermentação (Feitosa et al., 2010). O caldo fermentado, obtido com $144 \mathrm{~h}$ de 
fermentação, foi centrifugado à $3000 \mathrm{rpm}$ por 30 minutos e as proteínas foram precipitadas com $\left(\mathrm{NH}_{4}\right)_{2} \mathrm{SO}_{4}$ a $80 \%$ de saturação. A fase aquosa foi dialisada em membrana com massa de corte $12.000 \mathrm{Da}$ contra água ultra-pura durante 12 horas à $4^{\circ} \mathrm{C}$ e purificada em sistema aquoso bifásico (SAB) formado por $20 \%$ de Polietilenoglicol 8000, $18 \%$ de Fosfato de potássio, $6 \%$ de $\mathrm{NaCl}$ à $\mathrm{pH} 6$ e temperatura de $4^{\circ} \mathrm{C}$. A fase de fundo do $\mathrm{SAB}$, que continha a enzima foi dialisada e liofilizada à $-50^{\circ} \mathrm{C}$. Após esta etapa a enzima foi mantida em frasco fechado e mantida à $4^{\circ} \mathrm{C}$ (Barbosa et al., 2011).

\subsection{Imobilização da Enzima em PHBV na presença de LIs}

A enzima lipase Bacillus sp. (ITP-001) foi imobilizada por adsorção física de acordo a metodologia descrita por Cabrera-Padilla et al. (2013) com adição de 1\% (v/v) de líquido iônico. Foram utilizados os seguintes líquidos iônicos:

BI-LI1: Cloreto de 1- butil-3- metilpiridinio $\left(\left[\mathrm{C}_{4} \mathrm{mpy}\right] \mathrm{Cl}\right)$;

BI-LI2: Cloreto de 1- butil-3- metilimidazólio ([ $\left.\left.\mathrm{C}_{4} \mathrm{~min}\right] \mathrm{Cl}\right)$;

BI-LI3: Diacimida de 1- butil-3- metilimidazólio $\left(\left[\mathrm{C}_{4} \mathrm{~min}\right] \mathrm{N}(\mathrm{CN})_{2}\right)$;

BI-LI4: Bis(trifluorometilsulfonil)imida de 1-butil-3- metilimidazólio $\left(\left[\mathrm{C}_{4} \mathrm{~min} \mathrm{Tf}_{2} \mathrm{~N}\right)\right.$;

BI-LI5: Bis(trifluorometilsulfonil)imida de 1-Etil-3-metilimidazólio $\left(\left[\mathrm{C}_{2} \mathrm{~min}\right] \mathrm{Tf}_{2} \mathrm{~N}\right)$.

\subsection{Determinação da Atividade Hidrolítica e Recuperação da Atividade}

A atividade hidrolítica da lipase Bacillus sp. na forma livre e imobilizada foi determinada pelo método de hidrólise do azeite de oliva de acordo com o procedimento descrito por Soares et al. (1999), com modificações.

A recuperação da atividade (RA) foi determinado pela unidade de atividade hidrolítica total presente no suporte $\left(\mathrm{U}_{\mathrm{S}}\right)$ e unidades de atividade hidrolítica oferecida para imobilização $\left(U_{o}\right)$, como pode ser visto na Equação 1 .

$$
R A(\%)=\frac{U_{s}}{U_{o}} \times 100
$$

\subsection{Caracterização Bioquímica do Biocatalisador Imobilizado}

O biocatalisador imobilizado obtido utilizando LIs como aditivos que obteve o maior recuperação de atividade foi caracterizado quanto à:

Atividade em função do $\mathrm{pH}$ e da temperatura: Foi estudada utilizando-se azeite de oliva como substrato na faixa de $\mathrm{pH}$ entre 3,0 a 9,0. A influência da temperatura na atividade da LCR imobilizada foi determinada em $\mathrm{pH} 7,0$ nas temperaturas de 30 a $80^{\circ} \mathrm{C}$.

Estabilidade térmica: Foi determinado por meio da incubação do biocatalisador $(0,1 \mathrm{~g}$ massa seca) a 37 e $60^{\circ} \mathrm{C}$ em meio-aquoso (tampão fosfato $0,1 \mathrm{M}, \mathrm{pH} 4,0$ ) por 4 horas. Em tempos pré-estabelecidos, foram retiradas alíquotas e imediatamente resfriadas. Em seguida, a atividade hidrolítica residual foi determinada a $37^{\circ} \mathrm{C}(10 \mathrm{~min})$, como descrito anteriormente. 
Determinação dos parâmetros cinéticos: Para a determinação dos parâmetros cinéticos $\left(K_{m}\right.$ e $\left.V_{\max }\right)$ foram preparados sistemas reacionais contendo ácidos graxos totais em concentrações entre 37 e $2232 \mathrm{mM}$, obtidos a partir de emulsões preparadas com diferentes proporções de azeite de oliva (1 a 70\%) e solução aquosa de goma arábica ( $7 \%$ p/v). Os valores de $K_{m}$ e $V_{\max }$ aparentes foram calculados mediante ajuste não linear com o auxilio do programa Origin $^{\circledR} 8,0$.

Estabilidade operacional em meio aquoso: A estabilidade operacional e capacidade de reutilização do sistema imobilizada foram determinadas através da realização de reações de hidrólise em bateladas consecutivas, utilizando a mesma enzima imobilizada. Cada batelada consistia em uma reação de hidrólise durante 1 hora a uma temperatura de $37^{\circ} \mathrm{C}$ e $\mathrm{pH} 4,0$. Depois de cada batelada, a enzima imobilizada foi lavada com hexano e depois reutilizada para o próximo ciclo de hidrólise. Este procedimento foi repetido durante vinte ciclos. $\mathrm{O}$ tempo de meia vida $\left(\mathrm{t}_{1 / 2}\right)$ do biocatalisador foi determinado por aplicação do modelo de decaimento linear invertido (Da Rós et al., 2012).

\section{RESULTADOS E DISCUSSÃO}

\subsection{A atividade enzimática dos biocatalisadores imobilizados com líquidos iônicos.}

Os resultados da recuperação da atividade (RA) dos biocatalisadores imobilizados foram plotados na Figura 1. Todos os biocatalisadores imobilizados na presença de líquido iônico apresentaram maior RA comparado com o biocatalisador imobilizado sem LI (BI-SLI) $(\mathrm{RA}=25 \%)$.

Inicialmente, nossos resultados sugerem que a mudança da familia catiônica dos líquidos iônicos influemciam na RA (IB-IL1, IB-IL2); o maior valor foi encontrado para $\left[\mathrm{C}_{4} \mathrm{~min}\right] \mathrm{Cl} / \mathrm{LI}$ 2. Para os biocatalisadores imobilizados na presença de líquidos iônicos com o mesmo cation $\left[\mathrm{C}_{4} \mathrm{mim}\right]^{+}$e diferentes ânions (BI-LI2, BI-LI3, BI-LI4), a RA dos biocatalisadores seguiram a tendência $\mathrm{Tf}_{2} \mathrm{~N}^{-}>\mathrm{Cl}^{-}=\mathrm{N}(\mathrm{CN})_{2}{ }^{-}$. Valores semelhantes de RA foram obtidos para o BI-LI2 e BI-LI3 e menores do que o BI-LI4, provavelmente devido à natureza hidrofílica dos ânions $\mathrm{Cl}^{-} \mathrm{e} \mathrm{N}(\mathrm{CN})_{2}{ }^{-}$dos líquidos iônicos (Singh et al., 2010).

Para os biocatalisadores imobilizados na presença de líquidos iônicos, com o mesmo ânion $\mathrm{Tf}_{2} \mathrm{~N}^{-}$(BI-LI4, BI-LI5), observou-se um incremento da RA com o aumentou do comprimento da cadeia de substitutos do cátion dos líquidos iônicos. Assim, pode ser visto que a utilização de líquidos iônicos hidrófobicos influenciam positivamente no processo de imobilização, tal como evidenciado pelo aumento da recuperação da atividade dos biocatalisadores imobilizados com líquidos iônicos, [ $\left.\mathrm{C}_{4} \mathrm{~min}\right] \mathrm{Tf}_{2} \mathrm{~N} / \mathrm{LI} 4$ e $\left[\mathrm{C}_{2} \mathrm{~min}\right] \mathrm{Tf}_{2} \mathrm{~N} / \mathrm{LI} 5$ (Figura 1). O mais eficiente foi o biocatalisador imobilizado com o líquido iônico mais hidrofóbico [ $\left.\mathrm{C}_{4} \mathrm{~min}\right] \mathrm{Tf}_{2} \mathrm{~N}$ (BI-LI4) com RA de 56\%, mais do que o dobro do valor de BI-SLI. 


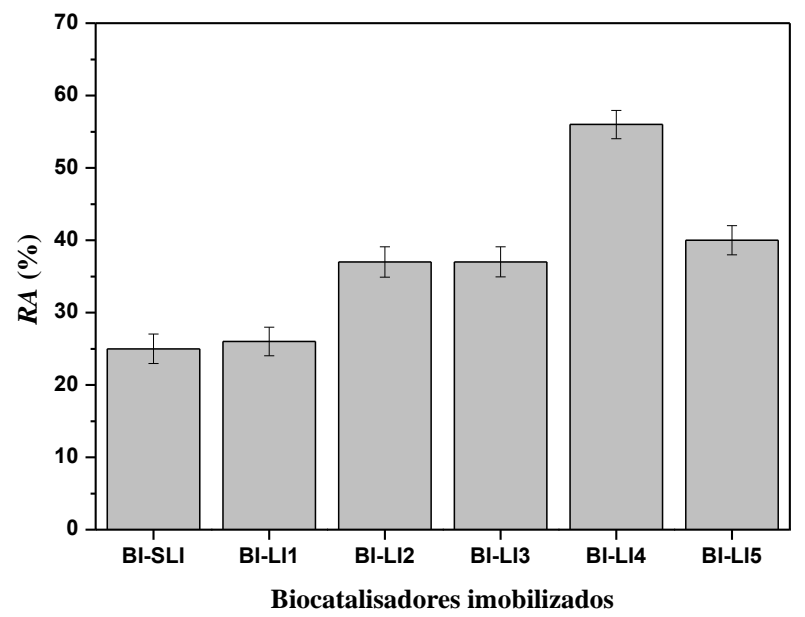

Figura 1 - Recuperação da atividade de lipase desde Bacillus sp. (ITP-001) imobilizada em PHBV na ausência e na presença de líquidos iônicos. As barras de erro são desvios-padrão de triplicatas.

\subsection{Caracterização Bioquímica do Biocatalisador Imobilizado}

Atividade em função do $\mathrm{pH}$ e da temperatura: Após a determinação do biocatalisador imobilizado na presença de $\left[\mathrm{C}_{4} \mathrm{~min}\right] \mathrm{Tf}_{2} \mathrm{~N} /(\mathrm{BI}-\mathrm{LI} 4)$ como o mais eficiente, foi verificado o efeito do $\mathrm{pH}$ do meio sobre a atividade das lipases imobilizadas, na ausência (BI-SLI) e presença (BI-LI4) de líquido iônico, para as reações de hidrólise na faixa de $3,0-9,0$ a $37^{\circ} \mathrm{C}$, os resultados são mostrados na Figura 2a. A adição de líquido iônico no processo de imobilização não causam mudança no $\mathrm{pH}$ óptimo para a atividade, de fato as maiores atividades obtidas por ambos os biocatalisadores imobilizados foi na faixa de 3,0-4,0, com o pH otimo 4,0. Foram observados perfis semelhantes para os biocatalisadores imobilizados sem e com LI na faixa de $\mathrm{pH}$ estudada, sendo ligeiramente mais estável para o biocatalisador imobilizado com o líquido iônico $\left[\mathrm{C}_{4} \mathrm{~min} \mathrm{Tf}_{2} \mathrm{~N} /(\mathrm{BI}-\mathrm{LI} 4)\right.$.

O efeito da temperatura na faixa de $30-80{ }^{\circ} \mathrm{C}$, sobre a atividade da enzima a $\mathrm{pH} 4$ é mostrada na Figura 2b. Os biocatalisadores imobilizados sem (BI-SLI) e com presença (BILI4) de líquidos iônicos apresentam perfis semelhantes, mantendo-se ambos praticamente constantes ao longo da faixa estudada. Assim, como o $\mathrm{pH}$, a temperatura óptima não sofre deslocamento com a adição de líquidos iônicos no processo de imobilização, para ambos os biocatalisadores imobilizados (BI-SLI e BI-LI4), a temperatura óptima para atingir a maior atividade foi de $37^{\circ} \mathrm{C}$. No entanto, foi observado que BI-LI4 foi ligeiramente mais resistente ao calor, na maioria das temperaturas estudadas, o que pode ser atribuído à ligação de hidrogênio e a interação eletrostática entre líquido iônico e enzima que gerou uma elevada barreira cinética para o desdobramento da enzima, deste modo, a estrutura rígida da enzima foi protegida de ser destruída (Zou et al., 2010). 

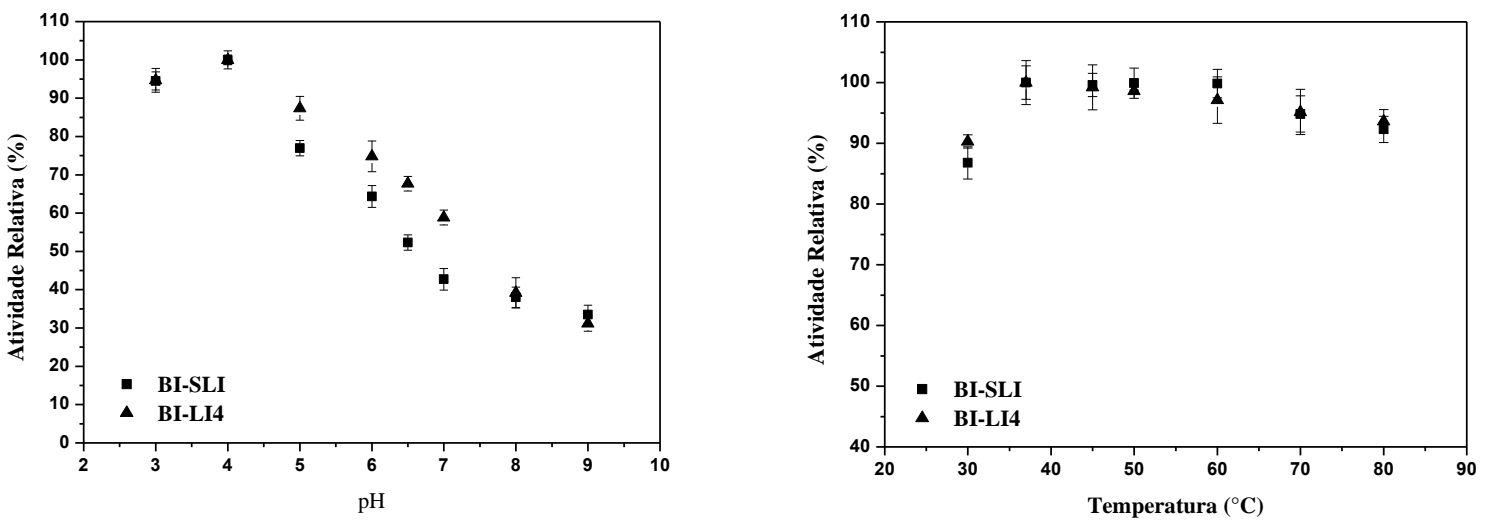

Figura 2 - Efeito do $\mathrm{pH}$ e temperatura na atividade relativa dos biocatalisadores imobilizados sem (BI-SLI) e com adição de liquido iônico (BI-LI4). As barras de erro são desvios-padrão de triplicatas.

Estabilidade térmica: A estabilidade térmica dos biocatalisadores imobilizados sem (BI-SLI) e com líquido iônico [ $\mathrm{C}_{4}$ min] $\mathrm{Tf}_{2} \mathrm{~N} /(\mathrm{BI}-\mathrm{LI}$ 4) foi determinada através da medição da atividade residual em função do tempo às temperaturas de $37^{\circ} \mathrm{C}$ e $60^{\circ} \mathrm{C}$ e são mostradas na Figura 3.

Como podem ser observados ambos os biocatalisadores imobilizados apresentam um comportamento semelhante para as duas temperaturas e se mantêm praticamente constantes durante todo o período de tempo estudado. Além disso, verificou-se que a adição de líquidos iônicos no processo de imobilização não influência significativamente na melhoria da estabilidade térmica.

No entanto, a literatura sugere que os líquidos iônicos podem preservar a conformação compacta da enzima, e reduzir a sua taxa de destruição da estrutura secundária a uma temperatura elevada (Rantwijk et al., 2006).

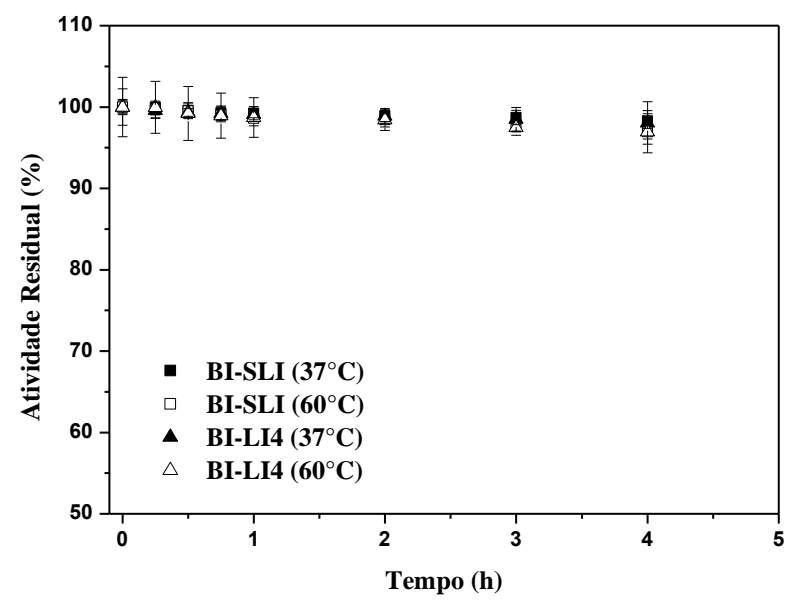

Figura 3. Estabilidade térmica para os biocatalisadores imobilizados sem (BI-SLI) e com adição de líquido iônico (BI-LI4) (incubadas em 37 e $60^{\circ} \mathrm{C}$ ). As barras de erro são desviospadrão de triplicatas. 
Determinação dos parâmetros cinéticos: A cinética do biocatalisador imobilizado com adição de líquido iônico (BI-LI4) foi investigada em várias concentrações de substrato a base de aceite de oliva (a $37^{\circ} \mathrm{C}, \mathrm{pH} 4,0$ ). A equação de Michaelis-Menten foi usada para ajustar os parâmetros cinéticos, $K_{m}$ e $V_{\max }$, com ajuda do programa Origin®. O $K_{m}$ e $V_{\max }$ do biocatalisador imobilizado foram 73,5 $\mathrm{mM}$ e $253,5 \mathrm{U} / \mathrm{g}$, respectivamente. Estes valores obtidos para as constantes cinéticas foram maiores comparadas com as obtidas para $\mathrm{o}$ biocatalizador imobilizado em ausencia de líquidos iônicos (BI-SLI) $\left(K_{m}=49,1 \mathrm{mM}\right.$ e $V_{\max }=$ $205 \mathrm{U} / \mathrm{g})$, essas mudanças nos parâmetros cinéticos $\left(K_{m}\right.$ e $\left.V_{\max }\right)$ sugerem que o líquido iônico $\left.\left(\left[\mathrm{C}_{4} \mathrm{~min}\right] \mathrm{Tf}_{2} \mathrm{~N}\right]\right)$ oferece um ambiente que aumenta a disponibilidade do sítio ativo da enzima com o substrato.

Estabilidade operacional: A reutilização das enzimas imobilizadas é muito importante para a sua aplicação, especialmente em aplicações industriais. No presente trabalho, a estabilidade do sistema imobilizada foi também avaliada através da reutilização do biocatalisador imobilizado na reação de hidrólise. A Figura 4 mostra a atividade residual em função do tempo de operação a partir da qual pode ser observada uma lenta diminuição na atividade de hidrólise com uma redução global de $27 \%$ no final do vigésimo reciclo, revelando um tempo de meia-vida $\left(\mathrm{t}_{1 / 2}\right)$ de 74,85 $\pm 0,69$ horas. Este resultado é superior ao estimado para esta lipase imobilizada, na ausência de líquido iônico, o que mostrou que, após dez ciclos (1h), a enzima reteve $33 \%$ da sua atividade inicial (Cabrera-Padilla et al., 2013).

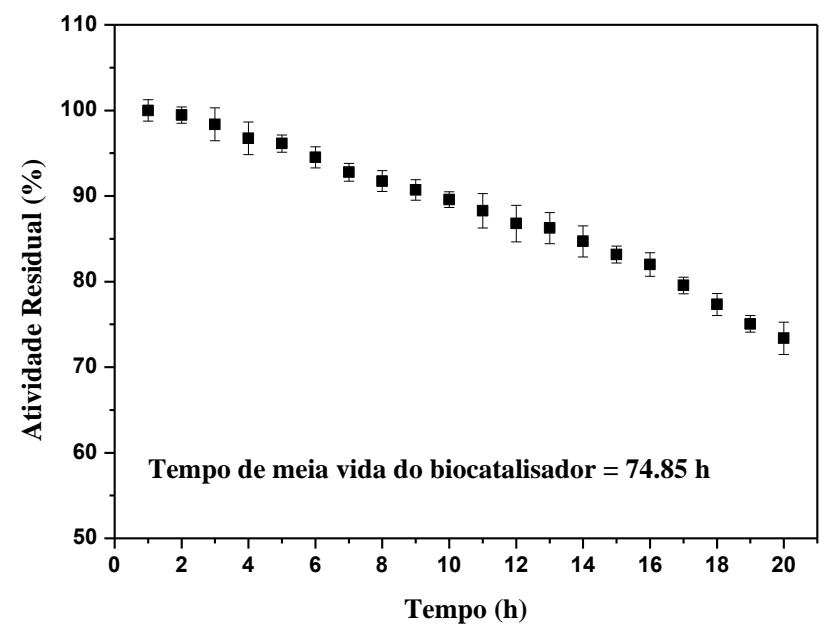

Figura 4 - Variação na atividade relativa, com o uso repetido do biocatalisador imobilizado

(BI-LI4). As barras de erro são desvios-padrão de triplicatas.

\section{CONCLUSÃO}

Neste estudo foi realizada a imobilização da lipase Bacillus sp.(ITP-001) com adição de líquido iônico. Os melhores resultados foram obtidos para os LIs hidrofóbicos $\left(\left[\mathrm{C}_{2} \mathrm{~min}\right] \mathrm{Tf}_{2} \mathrm{~N}\right.$ e $\left[\mathrm{C}_{4} \mathrm{~min}\right] \mathrm{Tf}_{2} \mathrm{~N}$ ) com uma recuperação total da atividade de $40 \%$ e $56 \%$, respectivamente. Além 
disso, o BI com LI ([ $\left.\left.\mathrm{C}_{4} \mathrm{~min}\right] \mathrm{Tf}_{2} \mathrm{~N}\right)$ apresentou uma consideravel melhora na capacidade de reutilização comparado com o biocatalizador imobilizado sem líquido iônico (BI-SLI).

\section{REFERÊNCIAS}

Barbosa, J. M. P.; Souza, R. L.; Fricks, A. T.; Zanin, G. M.; Soares, C. M. F.; Lima, A. S. Purification of lipase produced by a new source of Bacillus in submerged fermentation using an aqueous two-phase system. J. Mol. Catal. B-Enzym., v. 879, p. 3853-3858, 2011 .

Cabrera-Padilla, R. Y.; Lisboa, M. C.; Fricks, A. T.; Franceschi, E.; Lima, A. S.; Silva, D. P.; Soares, C. M. F. Immobilization of Candida rugosa lipase on poly(3-hydroxybutyrateco-hydroxyvalerate): a new eco-friendly support. J. Ind. Microbiol. Biotechnol., v.39, p. 289-298, 2011.

Cabrera-Padilla, R. Y.; Albuquerque, M.; Figueiredo, R. T.; Fricks, A. T.; Franceschi, E.; dos Santos, O. A. A.; Lima, A. S.; Silva, D. P.; Soares, C. M. F. Immobilization and characterisation of a lipase from a new source, Bacillus sp. ITP-001. Bioprocess Biosys. Eng., v. 36(10), p. 1385-1394, 2013.

Da Rós, P. C. M.; de Castro, H. F.; Carvalho, A. K. F.; Soares, C. M. F.; de Moraes, F. F.; Zanin, G. M. Microwave-assisted enzymatic synthesis of beef tallow biodiesel. J. Ind. Microbiol. Biotechnol., v. 39, p. 529-536, 2012.

Feitosa, I. C., Barbosa, J. M. P., Orelana, S. C, Lima, A. S, Soares C. M. F. Produção de lipase por meio de microrganismos isolados de solos com histórico de contato com petróleo. Acta Sci Technol., v. 32, p. 27-31, 2010.

Guncheva, M.; Zhiryakova, D. Catalytic properties and potential applications of Bacillus lipases. J. Mol. Catal. B-Enzym., v. 68, p. 1-21, 2010.

Hara, P.; Mikkola, J. P.; Murzin, D. Y.; Kanerva, L. T. Supported ionic liquids in Burkholderia cepacia lipase-catalyzed asymmetric acylation. J. Mol. Catal. B-Enzym., v. 67, p. 129-134, 2010.

Lee, S. H.; Doan, T. T.N.; Ha, S. H.; Koo, Y. M. Influence of ionic liquids as additives on sol-gel immobilized lipase. J. Mol. Catal. B-Enzym., v. 45, p. 57-61, 2007.

Mendes, A. A.; Oliveira, P. C.; Vélez, A. M.; Giordano, R. C.; Giordano, R. L. C.; de Castro, H.F. Evaluation of immobilized lipases on polyhydroxybutyrate beads to catalyze biodiesel synthesis. Int. J. Biol. Macromol., v. 50, p. 503- 511, 2012.

Rantwijk, V. F.; Secundo, F.; Sheldon, R. A. Structure and activity of Candida antarctica lipase B in ionic liquids. Green Chem., v. 8, p. 282-286, 2006.

Singh, N. R.; Narinesingh, D.; Singh, G. Immobilization of $\beta$-galactosidase onto Sepharose and stabilization in room temperature ionic liquids, J. Mol. Liq., v. 152, p. 19-27, 2010.

Soares, C. M. F.; Castro, H. F.; Moraes, F. F.; Zanin, G. M. Characterization and utilization of Candida rugosa lipase immobilized on controlled pore silica. App. Biochem. Biotechnol., v. 77-79, 745-758.

Zou, B.; Hu, Y.; Yu, D.; Xia, J.; Tang, S.; Liu, W.; Huang, H. Immobilization of porcine pancreatic lipase onto ionic liquid modified mesoporous silica SBA15. Biochem. Eng. J., v. 53, p. 150-153, 2010. 